\section{Clearing the path to Wnt}

\section{By Steve Edelson, Executive Editor}

Despite the clear involvement of the Wnt pathway in cancer, ${ }^{1,2}$ there are no disclosed inhibitors of Wnt signaling in the clinic. The reasons are twofold: the pathway is almost entirely intracellular and most of the interactions are protein-protein. Thus, antibodies are a poor option because they do not readily get inside of cells, and small molecules are equally challenging because they are not good at blocking proteinprotein interactions.

Now, a team at Novartis AG's Novartis Institutes for Biomedical Research (NIBR) has found two new components of the Wnt pathway-TNKS1 (tankyrase TRF1-interacting ankyrin-related ADP-ribose polymerase) and TNKS2 - that could be more druggable because they are enzymes. The company already has small molecule tankyrase inhibitors in earlystage development.

The tankyrases were found to degrade axin, which is part of a complex that regulates $\beta$ -

\section{"We opted to find a} molecule that does what you want to see and used tools to work backward to how it works."

$$
\text { - Jeffery Porter, Novartis AG }
$$

pounds bound to and then identified the real targets. Our key contribution was the map to the needle."

"We didn't want to make assumptions about how the pathway works," Porter told SciBX. "Instead, we opted to find a molecule that does what you want to see and used tools to work backward to how it works. What we found was, not surprisingly, a molecule taking advantage of normal feedback loops and systems that cells can use to control $\beta$-catenin levels."

The small molecule XAV939 was found to inhibit the two tankyrase enzymes. In vitro, the compound significantly inhibited proliferation of colorectal cancer cells. Results were published in Nature. ${ }^{3}$

Although TNKS1 and TNKS2 have slightly different amino acid sequences, Porter said, "you actually need to take out both of them to have the best effects. There are clearly some redundant functions."

"Our study uncovers a new mechanism that controls axin protein stability and Wnt pathway signaling, and its therapeutic exploitation holds promise for treating Wnt pathway-dependent cancers," the Nature authors wrote.

"Hitting this enzymatic activity should be a lower barrier" than developing inhibitors of protein-protein interactions, Porter said. "We have early hits and leads and we want to better understand the biology around the target."

He said the pharma's ongoing work involves fleshing out the mechanism of the tankyrase enzymes.

In addition to Novartis, a handful of companies have programs interrogating the Wnt pathway. Clinical Data Inc. gained a small molecule inhibitor of $\beta$-catenin last year when catenin, the key transcription factor in the Wnt pathway. When the complex malfunctions in many cancers, including about $80 \%$ of colorectal cancers, $\beta$-catenin is unchecked and causes aberrant expression of many genes in the Wnt pathway.

"In oncology, the most validated connection is in colon cancer. In that condition, you've lost control of the Wnt pathway down the line at the transcription factor stabilization step," said Jeffery Porter, an author on the paper and global head of developmental and molecular pathways at NIBR. "There's just been a real challenge to find druggable targets."

Instead of an arduous search for targets against which to develop small molecules, the researchers did the opposite-they threw small molecules at the Wnt pathway and worked backward to find out what the molecules hit.

The research is rooted in Novartis' 2004 collaboration with Cellzome Inc. to identify protein targets in various disease areas. The deal included Cellzome's chemical proteomics technology, in which small molecules are chemically altered to attach to a matrix and then are exposed to diseased cells to find proteins the small molecules bind.

"Novartis did the cell-based drug screens and went through their library of Wnt pathway modulators, but that was like looking for a needle in a haystack," said Cellzome CSO David Simmons. "We made linkable versions of those molecules and fished out what those com- it acquired Avalon Pharmaceuticals Inc. for $\$ 10$ million in stock. The compound, AVN316, is in preclinical development for cancer.

In 2007, Pfizer Inc. and one of its incubator companiesWintherix LLC-partnered to use Wintherix's biological knowledge and Pfizer's compound library to search for molecules that inhibit Wnt-related signaling pathways in cancer cells.

Novartis has filed a patent application covering the findings described in Nature.

Edelson, S. SciBX 2(37); doi:10.1038/scibx.2009.1397

Published online Sept. 24, 2009

\section{REFERENCES}

1. Polakis, P. Curr. Opin. Genet. Dev. 17, 45-51 (2007)

2. Barker, N. et al. Nat. Rev. Drug Discov. 5, 997-1014 (2006)

3. Huang, S. et al. Nature; published online Sept. 17, 2009; doi:10.1038/nature08356

Contact: Feng Cong, Novartis Institutes for Biomedical Research, Cambridge, Mass.

e-mail: feng.cong@novartis.com

\section{COMPANIES AND INSTITUTIONS MENTIONED}

Cellzome Inc., Boston, Mass.

Clinical Data Inc. (NASDAQ:CLDA), Newton, Mass.

Novartis AG (NYSE:NVS; SIX:NOVN), Basel, Switzerland

Pfizer Inc. (NSYE:PFE), New York, N.Y.

Wintherix LLC, San Diego, Calif. 